\title{
Optimized polydopamine coating and DNA conjugation onto gold nanorods for single nanoparticle bioaffinity measurements
}

\author{
Marwa M. Azab ${ }^{\mathrm{a}, \mathrm{b}}$, Rédha Cherif c , Aryanne L. Finnie ${ }^{\mathrm{a}}$, Maha M. Abou El-Alamin ${ }^{\mathrm{b}}$, \\ Maha A. Sultan ${ }^{\mathrm{b}}$ and Alastair W. Wark*a \\ a Centre for Molecular Nanometrology, Technology and Innovation Centre, Dept. of Pure \& \\ Applied Chemistry, University of Strathclyde, 99 George St., Glasgow, G1 1RD, UK. \\ ${ }^{b}$ Analytical Chemistry Department, Faculty of Pharmacy, Helwan University, 11795, Cairo, Egypt. \\ ${ }^{c}$ Faculté de Chimie, Université Pierre et Marie Curie, 4 Place Jussieu, 75252 Cedex 05, France.
}

*Corresponding author: Email: alastair.wark@strath.ac.uk, Tel: +44 (0)141 548 3084, ORCID: orcid.org/0000-0001-8736-7566, ResearcherID: O-5400-2014 


\begin{abstract}
Gold nanorods (NRs) have attracted a great deal of interest for a variety of biomedical and sensing applications. However, developing robust methods for biofunctionalizing NRs has continued to be challenging, especially for NR-DNA conjugates. This is due to the presence of cetyltrimethylammonium bromide (CTAB), which plays an essential role in controlling the anisotropic particle growth. In this article, we systematically explore the growth of a polydopamine (PDA) layer on a range of NR surfaces, comparing different polyelectrolyte and alkanethiol coatings as well as direct CTAB displacement. This revealed that the PDA layer thickness and growth rate is strongly dependent on the underlying nanorod functionalization chemistry and allowed us to establish a preferred route for the creation of stable, non-aggregated suspensions of PDA-coated NRs. The utility of this platform was then demonstrated by self-assembling packed monolayers of single-stranded DNA on the outer surface. Both the surface attachment and bioactivity of the resulting NR-DNA conjugates was then demonstrated by performing bulk solution and single nanoparticle imaging fluorescence measurements.
\end{abstract}




\section{Introduction}

Gold nanorods (NRs) have attracted a great deal of interest for a variety of biomedical and sensing applications due to their highly tuneable optical properties over a wide visible-IR range in combination with high-yield approaches for their synthesis. ${ }^{1}$ However, developing robust methods for biofunctionalizing nanorods has continued to be challenging due to the initial presence of a surface bilayer of the surfactant, cetyltrimethylammonium bromide (CTAB), which has an essential role in the anisotropic particle growth. ${ }^{2} \mathrm{CTAB}$ is cytotoxic and has a medium affinity for the NR surface, with net desorption and destabilization of the colloid occurring when the bulk CTAB concentration drops below $\sim 1 \mathrm{mM} .^{3-5}$ As highlighted in recent reviews of nanorod conjugation chemistry, ${ }^{6,7}$ there are still relatively few examples of robust and facile routes to create NRs conjugated to single stranded DNA (ssDNA). The direct attachment of thiol-modified DNA onto NRs has been reported, ${ }^{8-11}$ however complete displacement of the oppositely charged CTAB is unlikely and improved approaches include the presence of surfactants and thiolated polyethylene glycol. ${ }^{11-14}$ Other alternatives involve first displacing CTAB with either an alkanethiol either directly ${ }^{15}$ or via a multi-step two phase extraction ${ }^{16,17}$ followed by DNA conjugation. The combination of encapsulation in a silica shell and silanization chemistry has also been reported. ${ }^{18}$ However, while polyelectrolyte coating is a widely-established method for NR functionalization, ${ }^{19}$ only electrostatic ssDNA surface immobilization has been described for such surfaces ${ }^{20}$ and attempts by us at covalent coupling of ssDNA onto coatings such as poly(acrylic acid) were unsuccessful. Given the potential utility of NR-ssDNA conjugates for a wide range of bioanalytical and assembly applications, there is still a need for simpler and more flexible approaches for NR functionalization.

Polydopamine (PDA) is a biopolymer formed by the polymerization of dopamine hydrochloride (DA) in alkaline conditions. Initially inspired by adhesive proteins in mussels ${ }^{21}$ PDA coating formation has been demonstrated as a valuable tool for the surface modification of a wide range of metallic, glass, ceramic and polymer substrates. ${ }^{22}$ Furthermore, the subsequent PDA coated surface is reactive to primary amine and thiol groups, which provides a route for the direct modification of PDA surfaces with various biofunctional molecules, ${ }^{23}$ including DNA. ${ }^{24-26}$ While most studies involving PDA coating have focused on surface-immobilized substrates, there have been a small number of recent studies involving colloidal nanoparticles. In particular, for plasmonic systems, PDA layer growth on metallic nanospheres ${ }^{26-30}$ as well as nanorods ${ }^{31-36}$ have been described. For the latter NR studies, all involve partial CTAB displacement with the presence of a PEG derivative needed to be added to promote colloidal stability, which has implications for achieving packed 
ssDNA surfaces. Another aspect of PDA coating formation that has not been explored to any extent is the role of the underlying surface chemistry, especially on colloidal surfaces, where factors such as surface charge are likely to have a significant impact on the PDA layer thickness and growth kinetics as well as colloidal stability.

In this article, we systematically explore the growth of PDA layers on a variety of both positively and negatively charged gold NR surfaces and then apply this study to select a system upon which to demonstrate an optimized route for creating high-density ssDNA monolayers on the PDA-NRs. The differently charged NR surfaces were prepared using various polyelectrolyte chemistries with the changes in localized surface plasmon resonance (LSPR) used to monitor PDA layer growth and dependence on parameters such as dopamine concentration and reaction time. This study enabled us to establish a preferred route for the preparation of PDA-coated nanorods that could be subsequently used as a substrate for the bioconjugation of high density ssDNA monolayers. A combination of bulk fluorescence and single nanoparticle imaging measurements were then applied to confirm the surface attachment and also the bioactivity of the NR-ssDNA bioconjugates.

\section{Experimental}

Materials. Cetyltrimethylammonium bromide (CTAB) 99.0\%, hydrogen tetrachloroaurate $\left(\mathrm{HAuCl}_{4}\right) 99.9 \%$, ascorbic acid, silver nitrate $\left(\mathrm{AgNO}_{3}\right) 99.99 \%$, sodium borohydride $\left(\mathrm{NaBH}_{4}\right)$, dopamine hydrochloride (99.0\%), trizma Hydrochloride (99.0\%), 11-mercapto-undecanoic acid (MUA), boric acid, Ethylene diamine tetracetic acid (EDTA), poly(sodium 4-styrenesulfonate) (PSS) MW 70,000, poly(diallyldimethylammonium chloride) (PDDA) MW 100,000, poly(acrylic acid) (PAA) MW 15,000, poly(allylamine hydrochloride) (PAH) MW 15,000, sodium chloride $(\mathrm{NaCl})$, sodium hydroxide $(\mathrm{NaOH})$ and hydrochloric acid $(\mathrm{HCl})$ were all purchased from SigmaAldrich and used as received. All solutions were prepared using Milli-Q deionized water. Singlestranded DNA sequences (HPLC purified) were purchased from Integrated DNA Technologies: $\mathrm{P}_{\mathrm{NH} 2}=5^{\prime} \mathrm{NH}_{2}$-carbon12-CGAAATCCAGACACATAAGCACGAACCGAA-3'; $\mathrm{P}_{\mathrm{NH} 2, \mathrm{CY} 5}=5$ ' $\mathrm{NH}_{2}$ carbon12-CGAAATCCAGACACATAAGCACGAACCGAA-Cy5-3'; T $\mathrm{NH}_{2}=$ 5' $\mathrm{NH}_{2}$-carbon12-

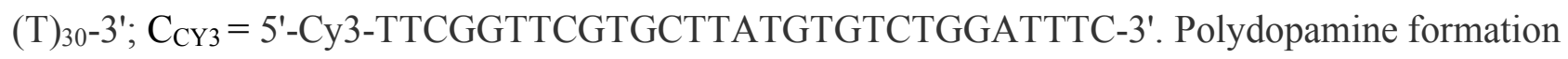
was performed by suspending various concentrations of dopamine hydrochloride in Tris buffer (10 $\mathrm{mM}$ Trizma) $\mathrm{pH}$ 8.5. The ssDNA conjugation to PDA was performed in TE buffer (10 mM Tris, 0.1 mM EDTA) pH 8.5. DNA hybridization was performed in PBS pH 7.2 (10 mM phosphate, $0.3 \mathrm{M}$ $\mathrm{NaCl})$. 
Nanorod preparation. Nanorod solutions were prepared via a scaled-up ( $450 \mathrm{~mL})$ version ${ }^{37}$ of the seed-based procedure developed by Nikoobakht and El-Sayed. ${ }^{38}$ Preparation of Seed Solution: A CTAB solution $(5 \mathrm{~mL}, 0.2 \mathrm{M})$ was mixed with $\mathrm{HAuCl}_{4}(5 \mathrm{~mL}, 0.82 \mathrm{mM})$ under stirring, to which freshly prepared $\mathrm{NaBH}_{4}(0.6 \mathrm{~mL}, 0.02 \mathrm{M})$ was added, giving a brownish-yellow solution. All CTAB containing solutions were kept in a water bath at $\sim 30^{\circ} \mathrm{C}$. Preparation of $A u$-NR's: NR's with a LSPR $\lambda_{\max }$ of $706 \mathrm{~nm}$ was obtained by adding $10 \mathrm{~mL}$ of $\mathrm{AgNO}_{3}$ solution (7.3 mg) to CTAB (200 $\mathrm{mL}, 0.2 \mathrm{M})$. Next, $\mathrm{HAuCl}_{4}(200 \mathrm{~mL}, 1 \mathrm{mM})$ was added and the solution gently mixed by inversion. Ascorbic acid $(2.8 \mathrm{~mL}, 0.08 \mathrm{M})$ was then added producing a color change from orange to colorless. Freshly prepared seed solution $(250 \mu \mathrm{L})$ was added to the growth solution, which was subsequently kept in a water bath at $\sim 30^{\circ} \mathrm{C}$ for $48 \mathrm{hrs}$. The solution was centrifuged at $8000 \mathrm{rpm}$ for 1 hour and re-suspended in $1 \mathrm{mM} \mathrm{CTAB}$ three times with the resulting stock solution stored in the water bath until use. Two batches of NR's were used with a LSPR $\lambda_{\max }$ at ca. 700 and $770 \mathrm{~nm}$ and whose concentrations were estimated using extinction coefficients of $3.6 \times 10^{9}$ and $4.5 \times 10^{9}$, respectively. ${ }^{39}$

Nanorod surface modification. Polyelectrolyte coating: A $10 \mathrm{~mL}$ solution of PSS $(10 \mathrm{mg} / \mathrm{mL})$ was added while dropwise under stirring to $40 \mathrm{~mL}$ of Au-NR's. After 5 mins, the colloidal suspension was centrifuged at $7500 \mathrm{rpm}(6290 \mathrm{~g})$ for 30 minutes and resuspended in water. A similar procedure was used for PDDA and PAH layers. Polydopamine film formation: Optimally, a $1 \mathrm{~mL}$ aliquot of $1.36 \mathrm{mM}$ dopamine $\mathrm{HCl}$ freshly dissolved in Tris buffer $\mathrm{pH} 8.5$ was added to a $3 \mathrm{~mL}$ aliquot of PSS-coated nanorods (O.D. $\sim 1$ at LSPR $\lambda_{\max }$ ). The reaction mixture was placed on an orbital shaker at room temperature for $4 \mathrm{hrs}$ with the solution color darkening due to PDA formation. The solution was then centrifuged at $9000 \mathrm{rpm}(9055 \mathrm{~g})$ for 10 minutes twice and re-suspended in water. A variety of dopamine $\mathrm{HCl}$ concentrations and reaction times were also explored as discussed later. The PDA film formation was also dependent on the shaking speed or the use of an ultrasound bath instead. MUA functionalization: CTAB was displaced on the NR surface, a $9.7 \mathrm{mM}$ MUA solution was prepared in $0.2 \mathrm{M} \mathrm{NaOH}$. To $5 \mathrm{~mL}$ of NR's resuspended in $0.5 \mathrm{mM} \mathrm{CTAB}, 4 \mathrm{~mL}$ of the MUA solution was added. The reaction mixture was placed in a ultrasound water bath at $55{ }^{\circ} \mathrm{C}$ for 30 mins and then for a further 3 hours at room temperature. The solution was centrifuged twice at 7800 rpm $(6800 \mathrm{~g})$ for $30 \mathrm{mins}$ and re-suspended in $0.01 \mathrm{M}$ borate buffer $\mathrm{pH}=9.2$.

DNA modification and hybridization: A $700 \mu \mathrm{L}$ aliquot of NR@PSS@PDA's were dispersed in TE buffer $\mathrm{pH}$ 7.2, to which $110 \mu \mathrm{L}$ of $15 \mu \mathrm{M}$ amine-terminated ssDNA ( $\mathrm{P}_{\mathrm{NH} 2, \mathrm{CY} 5}, \mathrm{P}_{\mathrm{NH} 2}, \mathrm{~T}_{\mathrm{NH} 2}$ ) was added and left to incubate at $37^{\circ} \mathrm{C}$. After leaving overnight, $1 \mathrm{M} \mathrm{NaCl}$ was added to increase the salt concentration to $150 \mathrm{mM}$. The reaction was left for another day and then centrifuged twice at 8400 rpm $(7890 \mathrm{~g})$ for 20 minutes and resuspended in TE buffer $\mathrm{pH} 7.2$ twice. Prior to DNA 
hybridization reactions, NR@PSS@PDA@DNA conjugates were dispersed in 0.3 M PBS buffer. Various concentrations of the complementary sequence (3-244 $\mathrm{nM})$ were then added to a fixed concentration of NR colloid (based on a maintained OD of 0.3 in the reaction volume) and left in the shaker for a minimum of 3 hours in order for the bioaffinity interaction to reach a steady-state.

Fluorescence and Imaging measurements: Bulk solution fluorescence measurements were acquired using a Cary Eclipse fluorescence spectrophotometer with samples in a $1 \mathrm{~cm}$ path-length 4-sided cuvette. Single NR reflection dark-field and fluorescence images were obtained using an upright Nikon Eclipse LV100 microscope, a 50x (NA = 0.55) ELWD objective and a CoolSNAP HQ monochrome camera $(0.01 \mathrm{~s}$ and $10 \mathrm{~s}$ integration times used for dark-field and fluorescence respectively). Polyelectrolyte coated glass cover slides were prepared, which were then exposed to a dilute solution of NR conjugates for $\sim 10$ mins before rinsing the surface gently with distilled water and drying in nitrogen. This created a relatively low surface coverage of NR's while minimizing potential aggregation. ${ }^{4}$ Scanning electron microscopy measurements were performed using a FEI Quanta 250 field-emission environmental scanning electron microscope (SEM), samples were prepared by exposing a polyelectrolyte (PDDA) coated Si wafer to the colloid solution for a few minutes before rinsing with water to prevent drying induced aggregation. ${ }^{4}$ Transmission electron microscopy (TEM) measurements were performed using a Jeol 1200 EXII operating at $80 \mathrm{kV}$.

\section{Results and Discussion}

\section{Optimizing polydopamine growth on NRs}

The first part of our investigation was to perform a systematic investigation of polydopamine (PDA) layer formation on different nanorod surfaces prior to DNA biofunctionalization. As summarized in Fig. 1, a variety of different underlying surface chemistries were explored. These include the wrapping of different combinations of polyelectrolytes in order to compare both positive and negative charged surfaces as the outermost layer prior to PDA formation. Both ourselves ${ }^{40}$ and others ${ }^{41}$ have previously demonstrated the high stability of polyelectrolyte wrapped nanorods, however, the cytotoxicity of CTAB is still a concern for potential in vivo applications and thus we also explored the displacement of CTAB either directly during the dopamine polymerization reaction or by first creating an 11-mercaptoundecanoic acid (MUA) monolayer on the NR surface, followed by PDA formation. 


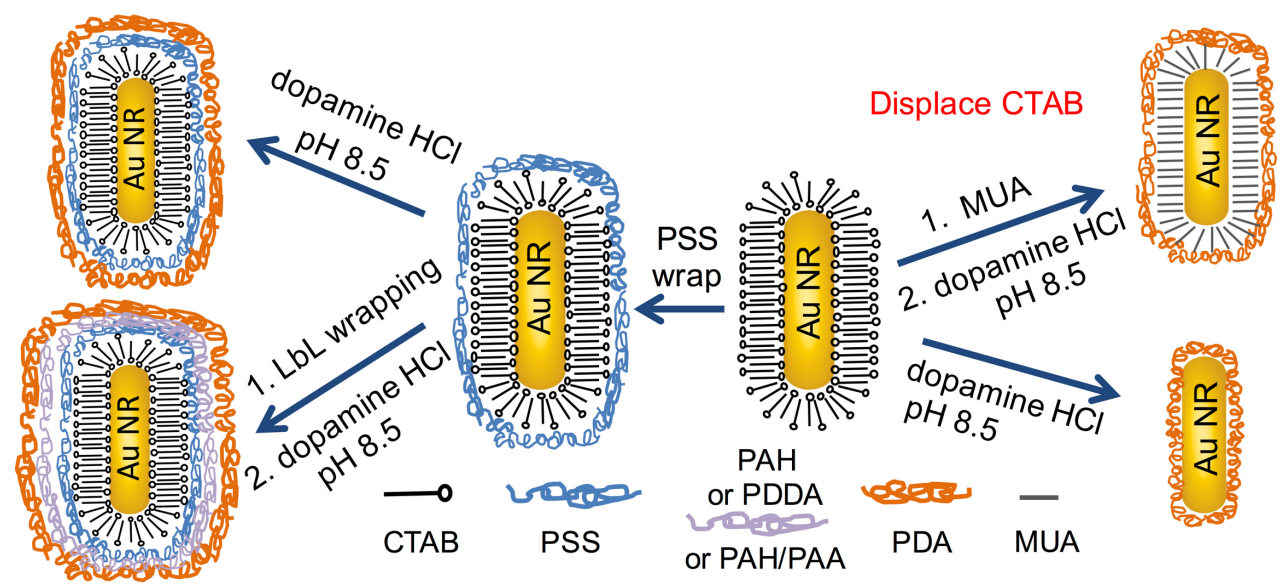

Fig. 1 Schematic summarizing the differently functionalized nanorod surfaces upon which the polydopamine (PDA) coating formation was monitored. This includes alternative outer layers formed by layer-by layer (LbL) assembly of either poly(sodium 4-styrenesulfonate) (PSS), poly(diallyldimethylammonium chloride) (PDDA), poly(acrylic acid) (PAA) or poly(allylamine hydrochloride) (PAH). Alternatively, CTAB was first displaced with 11-mercaptoundecanoic acid (MUA) prior to PDA layer growth.

\section{PDA growth on polyelectrolyte coated NRs}

In order to assess the effect of the outer molecular layer surrounding the nanorod surface on the polydopamine layer formation, a series of extinction spectra were acquired following different growth times. These results are summarized in Fig. 2 comparing PSS, PDDA, PAH or PAA as the outermost self-assembled layer formed prior to polydopamine growth (see ESI Fig. S1 for stock solution spectra). Experiments where surface adsorbed CTAB is (partially) displaced are discussed later. In each case, the initial nanorod and dopamine concentrations were kept the same $(0.2 \mathrm{nM}$ and $0.3 \mathrm{mM}$, respectively) with each spectrum representing a separate experiment where the reaction mixture was centrifuged and resuspended in water after various initial mixing times ranging from 5 mins to overnight. It is also important to note that all measurements in Fig. 2 were also performed at the same shaking speed during PDA growth and from the same batch of nanorods to compare the different LSPR shifts observed. For comparison, extinction spectra monitoring the $0.3 \mathrm{mM}$ dopamine polymerization in the absence of nanoparticles is shown in ESI Fig. S2.

The reaction conditions used in Fig. 2 were based on an initial set of measurements performed on PSS coated Au NRs. A typical approach was to choose a NR concentration with an OD in the $0.5-$ 1 range and then vary the dopamine concentration. As shown in ESI Fig. S3 relatively small changes in the bulk dopamine concentration between 0.1 and $0.5 \mathrm{mM}$ had a significant effect on the PDA layer growth rate. Attempts at working with higher dopamine concentrations were less successful due to difficulty in separating coated NRs from the reaction mixture. Consequently, for 
the remainder of this study we focused on a dopamine concentration of $0.3 \mathrm{mM}$ in order to achieve a controlled rate of polymer formation on different surfaces in a reasonable timeframe.
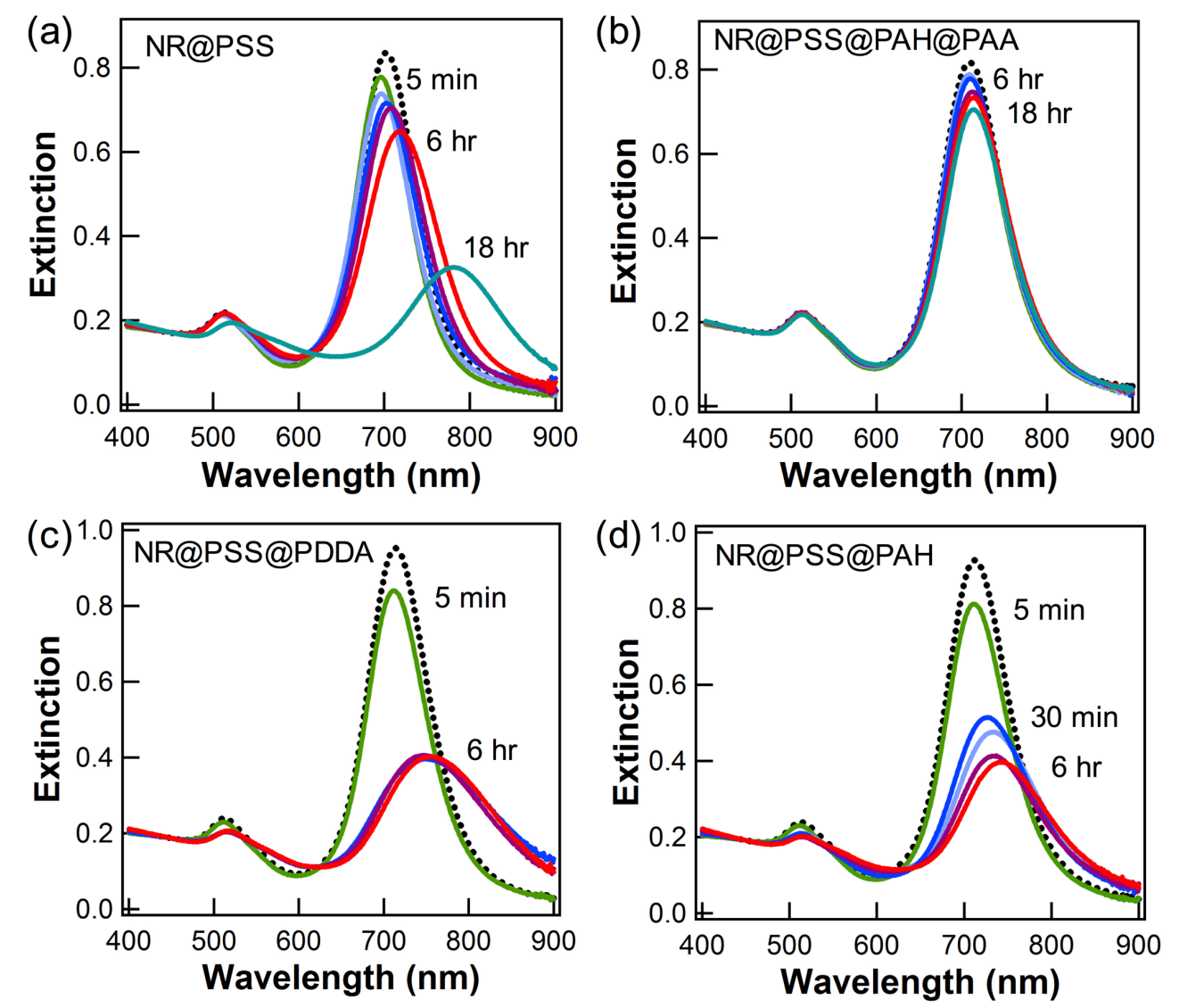

Fig. 2 Extinction spectra comparing PDA layer formation at different reaction times for (a) NR@PSS,(b)NR@PSS@PAH@PAA, (c)NR@PSS@PDDA and (d)NR@PSS@PAH.The black dashed curve represents the stock NR used for each data set. For each of the remaining spectra, the dopamine concentration was fixed at $0.3 \mathrm{mM}$ with various times of $5 \mathrm{~min}, 30 \mathrm{~min}, 1 \mathrm{hr}, 3 \mathrm{hr}, 6 \mathrm{hr}$, and $18 \mathrm{hr}$ representing the mixing time prior to centrifuging (for $2 \times 10 \mathrm{mins}$ ) and resuspending in water to halt further PDA layer growth. All spectra have been normalized to the same intensity at $450 \mathrm{~nm}$ for comparison purposes.

Looking first at PDA growth on NR@PSS in Fig. 2(a), the $\lambda_{\max }$ of the longitudinal LSPR peak at $702 \mathrm{~nm}$ undergoes an initial blue shift of $\sim 6 \mathrm{~nm}$ followed by an increasing red shift and damping as the PDA layer continues to grow at longer reaction times. After leaving overnight, a significant redshift of $80 \mathrm{~nm}$ was observed. The additional damping of the LSPR band observed can be potentially explained via either (i) controlled nanorod assembly or (ii) the electronic properties of the growing PDA layer. The observation of controlled blue-shifts for side-by-side ${ }^{4,42}$ and red-shifts for end-to-end ${ }^{42,43}$ or random nanorod assembly has been previously described. To confirm that the changes in the LSPR peak are associated with the electronic and refractive index properties of the PDA layer as opposed to NR assembly, nanoparticle tracking analysis (NTA) of colloidal solutions at some of the different PDA layer thicknesses shown in Fig. 2(a) was performed. The results 
shown in ESI Fig. S4 confirm an increase in the average particle size with longer reaction times. At 6 hours and overnight, the significantly increased average size and size distribution are affiliated with thicker PDA layer growth rather than a decrease in particle density due to partial aggregation. This is further supported by the representative SEM images of NR@PDA rods shown in Fig. 3(a) and ESI Fig. S5 which also indicated the NRs to be individually dispersed rather than aggregated.

Representative TEM images of NR@PSS@PDA samples are also shown in Fig. 3 and ESI Fig. S6. These measurements were performed on a separate batch of NRs with a LSPR $\lambda_{\max }$ similar to that in Fig. 2. No surrounding polymer film could be directly observed for the NR@PSS sample, however it was possible to determine the thicker PDA layer. For layers which exhibited a small LSPR red shift of $<10 \mathrm{~nm}$ (after 4-6 hours growth in $0.3 \mathrm{mM}$ ), a surrounding PDA layer thickness of $\sim 2-3 \mathrm{~nm}$ could be measured, while for a longer growth time ( $\sim 18$ hours) corresponding to a LSPR red shift of $\sim 15 \mathrm{~nm}$, a PDA shell thickness in the region of $\sim 5-6 \mathrm{~nm}$ was observed. These thickness values are lower than that reported by Wang et $a l^{26}$ on spherical Au citrate nanoparticles. However, a number of factors are important in layer growth rates in addition to the surface chemistry such as the dopamine : nanoparticle concentration ratio, buffer composition, mixing speed, temperature as well as reaction time.


(c)

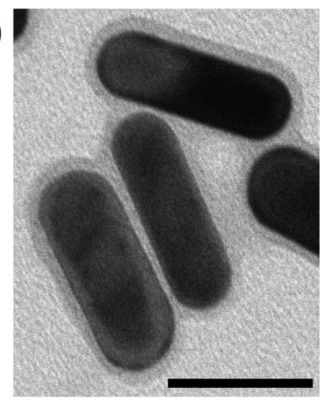

Fig.3 (a) Representative SEM image of NR@PSS@PDA prepared with6 hours of polymer growth time (see Fig. 2a) deposited on a PDDA-coated Si wafer and minimizing drying induced aggregation. Scale bar=500 nm.TEM images of separate NR@PSS@PDA samples with (c)4hrs and (d) 18 hrs growth times with the associated extinction spectra and additional images shown in ESI Fig. S6. Scale bars $=50 \mathrm{~nm}$. 
Comparing PDA layer growth on different polyelectrolyte coatings:

The data sets shown in Fig. 2(b), (c) and (d) where the respective outer polyelectrolyte layers are PAA, PDDA and PAH show significantly different PDA growth behaviors compared to that of NR@PSS in Fig. 2(a). This is highlighted by comparing the LSPR peak shifts in each case (plotted in ESI Fig. S7) which shows a much larger and more rapid change occurring for the positively charged NR@PSS@PAH and NR@PSS@PDDA samples compared to the negatively charged NR@PSS and NR@PSS@PAH@PAA substrates. Zeta-potential measurements were also acquired for samples prepared at various growth times (see ESI Table S1) where it was observed that a strongly negative zeta-potential was repeatedly measured irrespective of the corresponding PDA layer thickness or whether the NR was initially positively or negatively charged prior to PDA growth.

To enable comparison between each NR surface chemistry, the measurements in Fig. 2 were performed on the same orbital shaker at the same speed setting. One clear difference in behavior between the different samples is that the PDA layer growth (based on LSPR changes) is slower and more controlled on a negative charge surface (PSS and PAA), than on positive surfaces (PDDA) while the PAH layer is expected to be partially ionized at the buffer $\mathrm{pH}$. The molecular mechanism of polydopamine formation continues to be debated in the literature with various models proposed involving a series of intermediates and various polymerization, oxidation and interaction processes..$^{44}$ PDA layer formation has been demonstrated on a wide range of material substrates and the origin of this universality can be associated with the variety of functional groups created including indole units, amino, carboxylic acid, catechol or quinone, and $\pi$-systems that are incorporated into PDA. Cross-linking between monomer and polymer chain units can occur via several strong, non-covalent interactions (e.g. hydrogen bonding, $\pi$-stacking, charge transfer). Also, when combined with the low dopamine concentrations used here, it is feasible that noticeable differences in layer growth kinetics on different surfaces can be observed and the TEM measurements indicate that the nanorod LSPR is sensitive to sub-nm layer thickness changes. There are very few careful kinetic studies of PDA layer growth ${ }^{45}$ and we could not find any systematic studies of PDA growth on differently charged colloidal surfaces. Recent nanoparticle-based studies involving PDA layer formation have been mostly limited to citrate, ${ }^{26} \mathrm{CTAB},{ }^{32}$ or $\mathrm{PEG}^{29}$ stabilized colloids. Our results here clearly show that both the surface charge and surface functional group are just as important as the initial dopamine concentration.

\section{Comparing PDA growth on a different nanorod size:}

The size of the LSPR shifts described above during PDA growth will also depend on the electronic 
properties of the gold NRs. To explore this, a repeat set of data is shown in the ESI (Figs. S8 and S9) using the same conditions as applied in Fig. 2 except using a replacement batch of NRs featuring a longitudinal LSPR $\lambda_{\max }$ of $\sim 770 \mathrm{~nm}$ instead of $\sim 700 \mathrm{~nm}$. Comparing the PSS, PAH and PAA outer layers shows the same trend with more controlled PDA time-dependent growth on the PSS surface. However, the magnitude of the LSPR change is significantly different with a larger initial blue shift of 12 nm observed for the longer PSS@NR substrates (compared to 4 nm in Fig. 2a). It is well-established that LSPR sensitivity to local changes in refractive index is greater for longer aspect ratio $\mathrm{NRs}^{46}$ and for non-absorbent layers a greater red-shift would actually be expected for a similar PDA layer thickness. The fact that the opposite trend is observed here is attributed to the electronic properties of the PDA layer formed rather than significant differences in the layer thickness. The extinction spectra in ESI Fig. S2 shows that the PDA growth solution becomes increasingly absorbent across the visible wavelengths and the degree of spectral overlap between the NR LSPR and PDA layer will have a significant impact on the degree of electronic coupling and subsequent LSPR shift that is observed. As a comparison, this behavior has been studied for different NR-dye conjugate combinations. ${ }^{47,48}$

\section{PDA coating of NRs involving CTAB displacement.}

In addition to monitoring PDA formation on polyelectrolyte coated NRs, comparison measurements were also performed directly with CTAB stabilized NR stock solutions as well as first displacing the CTAB bilayer with 11-mercaptoundecanoic acid (MUA). In addition to extending the study by providing an alternative surface charge and functionalization for comparison, another motivation is the concern regarding the toxicity of CTAB although it has been demonstrated that the cytotoxicity of polymer encapsulated NRs is very low. ${ }^{37}$ Fig. 4(a) shows a typical result for PDA formation on CTAB stabilized NRs utilizing the same NR and dopamine concentrations as in Fig. 2(a). It can clearly be seen from the extinction spectra that aggregation is occurring at a longer reaction time of $1 \mathrm{hr}$, while the sample after 30 mins PDA growth remained fairly disperse after centrifugation and resuspension. However, the long-term stability of NR@PDA rods prepared in this manner was consistently poor, as shown in ESI Fig. S10 where initially stable samples would aggregate over a couple of days. In comparison, NR@PSS@PDA colloids had excellent long-term stability of several months (ESI Fig. S10). The presence of freely suspended positively charged CTAB during the PDA formation clearly has a destabilizing effect and previous reports of PDA coating of NR' ${ }^{32-}$

${ }^{34}$ all involve the introduction of PEG to prevent this. However, this also reduces the surface density of available attachment sites for the subsequent conjugation of active molecules onto the nanoparticle surface. 
In addition, we also explored the complete displacement of CTAB with an alkanethiol monolayer terminated with a carboxylic acid group (MUA) prior to PDA layer growth. After preparing stable NR@MUA conjugates (see ESI Fig. S11), PDA growth was performed under the same reaction conditions as above. As can be observed in Fig. 4(b), a red shift in the LSPR $\lambda_{\max }$ of $\sim 13 \mathrm{~nm}$ is observed after an hour and following this the peak position changes only slightly but damping is continued to be observed. This behavior is different compared to the negatively charged surfaces in Fig. 2 (i.e. PSS and PAA outer layers), and further highlights that both the functional groups and surface charge on the NR have an effect on the PDA layer growth.
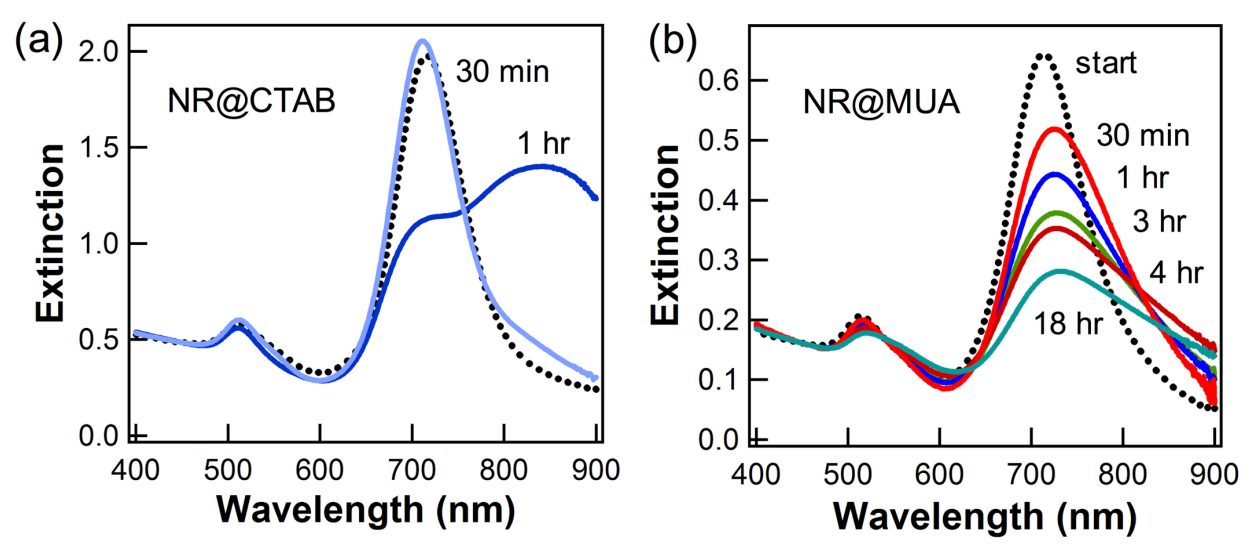

Fig. 4 Extinction spectra monitoring PDA coating at different reaction times for (a) CTAB stabilized NR's and (b) MUA stabilized NR's. The dashed curve represents the initial stock NR$M U A$ conjugates with the remaining curves obtained following centrifugation and resuspension in water after different PDA reaction times. The dopamine concentration was $0.3 \mathrm{mM}$ in both cases and in (a) the bulk CTAB concentration was reduced to $1 \mathrm{mM}$ prior to dopamine addition. All spectra have been normalized to the same intensity at $450 \mathrm{~nm}$ for comparison purposes.

\section{DNA conjugation to NR@PDA.}

Having demonstrated both controlled PDA growth and long-term stability on NR@PSS substrates, this platform was used to explore the formation of packed ssDNA monolayers as well as confirm the bioactivity of the resulting NR-ssDNA conjugates. This is outlined in Fig. 5 where the NR@PSS@PDA conjugates are incubated with ssDNA sequences that are 5' modified with a primary amine (for conjugation to PDA). A carbon12 spacer was selected to promote efficient conjugation rather than a polynucleotide spacer as both PDA and DNA are negatively charged; which also promotes the DNA orientation away from the NR surface. At $\mathrm{pH} 8.5$, catechol surface groups are oxidized to $o$-quinone intermediates which spontaneously undergo covalent crosslinking to nucleophilic 5' amines via a Michael addition and/or Schiff base reaction. ${ }^{26,49,50}$ This chemistry does have an advantage compared to using thiol-modified oligos for nanoparticle modification due to less handling required regarding long-term storage and disulfide bond cleavage prior to use. To monitor the ssDNA surface attachment onto the NR surface, a Cy5 dye molecule 


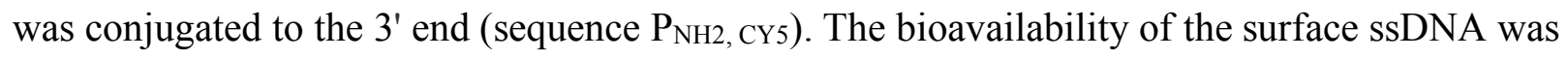
then monitored through exposure to the complementary sequence $\left(\mathrm{C}_{\mathrm{CY} 3}\right)$ which is modified with a Cy3 dye molecule and results in the creation of a fluorescence resonance energy transfer (FRET) pairing.
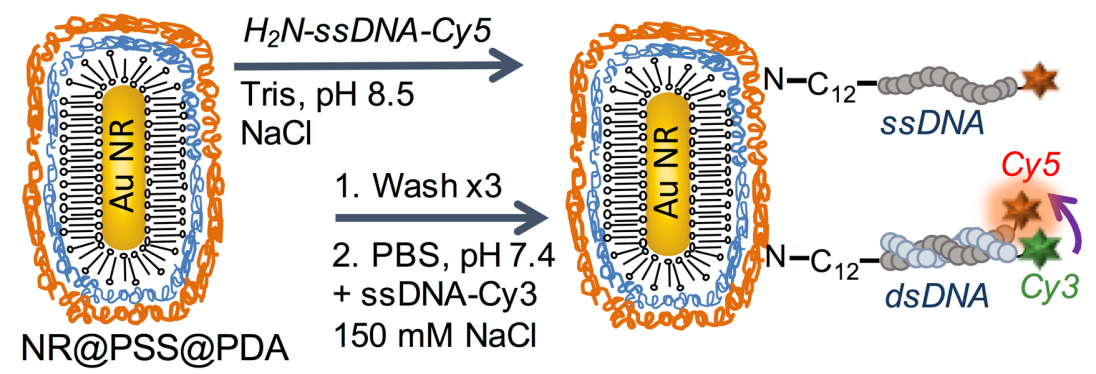

Fig. 5 Schematic overview of the steps for the covalent coupling of SSDNA to PDA coated NRs and subsequent hybridization reaction performed to confirm the bioavailability of surface immobilized sequences.

In a first set of experiments, solution fluorescence measurements were applied to quantify the conjugation of the $\mathrm{P}_{\mathrm{NH} 2, \mathrm{CY} 5}$ sequence onto the NR surface. Fig. 6(a) shows an example set of data where the intensity of the Cy5 fluorescence spectrum is compared before and after NR conjugation, with the NR-P $\mathrm{P}_{\mathrm{NH} 2, \mathrm{CY} 5}$ conjugates separated via centrifugation prior to analysis of the supernatant. Measurements were performed both with and without the presence of $0.15 \mathrm{M} \mathrm{NaCl}$ when preparing the conjugates to demonstrate the higher ssDNA surface densities that can be obtained by the charge-screening effects of the salt. Initial conjugation measurements were performed in the presence of a higher $(1.1 \mu \mathrm{M})$ ssDNA concentration. This corresponds to a loading ssDNA/NR ratio of $\sim 10,500$, which was chosen to promote maximum surface coverage. ${ }^{26,51}$ Our measurements (ESI Fig. S12 shows a linear fluorescence signal calibration curve) revealed an estimate of $\sim 1300( \pm 90)$ ssDNA strands per NR (LSPR $\lambda_{\max } 700 \mathrm{~nm}$ ). This is considerably higher than that reported by Wiyama et $a{ }^{10}$ who reported $\sim 120-230$ ssDNA strands per NR using an alkanethiol ligand exchange approach for surface functionalization and a similar number $(<300)$ is theoretically predicted by Hill et $\mathrm{al}^{52}$ though this model was only experimentally verified using much larger nanorod structures. In the absence of $\mathrm{NaCl}$, the ssDNA loading density was repeatedly ca. 45-50\% lower (along with a smaller LSPR peak change, Fig. 6b) than that achieved in the presence of 0.15 $\mathrm{M} \mathrm{NaCl}$, as expected. In addition, when comparing ssDNA conjugation between two different PDA layer thickness (formed at 4 and $18 \mathrm{hrs}$ growth) only a $\sim 10 \%$ difference in loading density was observed (data not shown) with the thicker PDA layer associated with a slightly higher ssDNA surface coverage. 
(a)

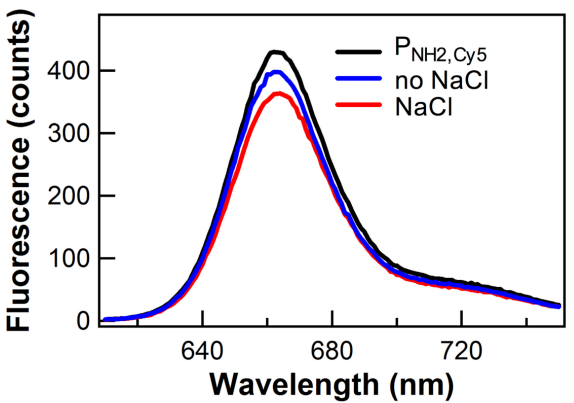

(c)

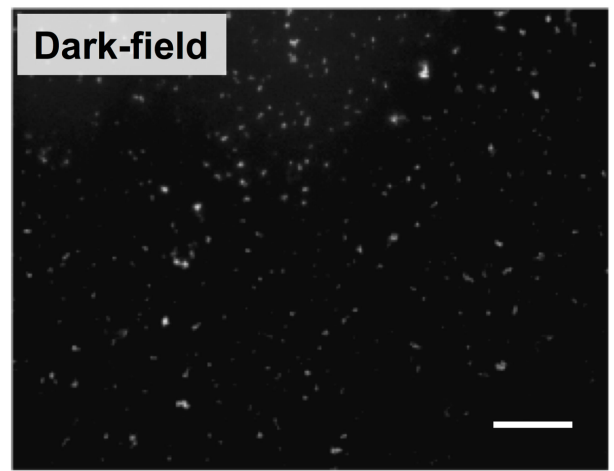

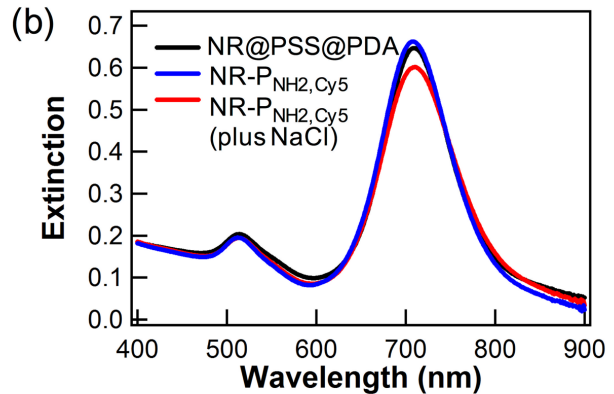

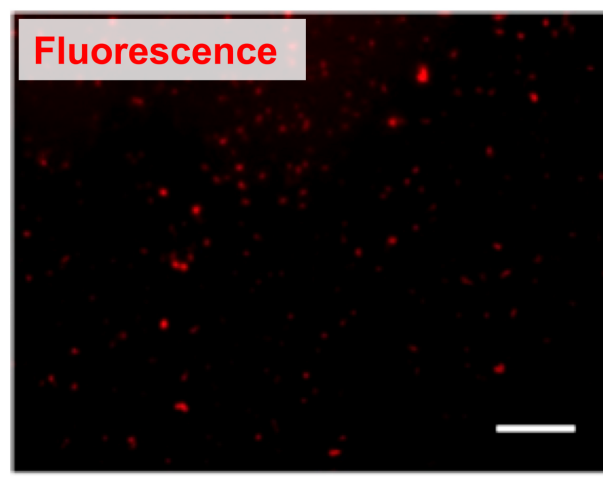

Fig.6 Monitoring ssDNA conjugation to NR@PSS@PDA's. (a) Fluorescence spectra ( $\lambda_{e x}=600$ $n m)$ demonstrating drop in Cy5 concentration associated with the NR-ssDNA formation. The initial $P_{N H 2, C Y 5}$ solution diluted to the reaction volume is compared to supernatant solutions obtained both in the presence and absence of $0.15 \mathrm{M} \mathrm{NaCl} \mathrm{following} \mathrm{conjugation.} \mathrm{(b)} \mathrm{Extinction} \mathrm{spectra} \mathrm{of}$ NR@PSS@PDA before and after $P_{\mathrm{NH} 2, \mathrm{CY} 5}$ conjugation. (c) Correlated dark-field and fluorescence images of $P_{\mathrm{NH} 2 \mathrm{CY} 5}$ conjugated $N R$ 's (in $0.15 \mathrm{M} \mathrm{NaCl}$ ). Scale bar $=16 \mu \mathrm{m}$.

It is well-established that the highest ssDNA packing occurs on smaller radius particles with densities as high as $\sim 250$ strands/particle reported for $15 \mathrm{~nm}$ particles $\left(0.35\right.$ strands per $\left.\mathrm{nm}^{2}\right)$ when PEG spacers are utilized instead of additional nucleotides. ${ }^{51}$ The NRs used for the DNA conjugation measurements have an aspect ratio of $\sim 2.8$ (LSPR $\lambda_{\max } \sim 700 \mathrm{~nm}$ ) and width of $17 \mathrm{~nm}$ and the neutral carbon-12 spacer used here should also facilitate higher packing. Estimating the thickness of the surrounding polymer layer as $\sim 4 \mathrm{~nm}$ (i.e. $25 \mathrm{~nm}$ width, $56 \mathrm{~nm}$ length) results in an estimate of 0.29 ssDNA strands per $\mathrm{nm}^{2}$ (at the high $\sim 1300$ per NP loading ratio). For comparison, on PDA coated Au nanospheres $\sim 50 \mathrm{~nm}$ in size, a DNA loading of $\sim 2050$ per NP ( 0.15 strands per $\mathrm{nm}^{2}$ considering PDA layer thickness) has been reported elsewhere. ${ }^{26}$ The higher DNA surface density observed here on PDA@NR's is approaching that reported for DNA-SH self-assembly methods on smaller Au nanospheres. ${ }^{51}$ Furthermore, the imaging of NR-P $\mathrm{P}_{\mathrm{NH} 2, \mathrm{CY} 5}$ conjugates immobilized on a glass slide in Fig. 6(c) also highlights the high ssDNA surface density and uniformity across the nanoparticle population with comparison of the dark-field and fluorescence images indicating over $>95 \%$ of features in the dark-field image exhibiting significant Cy 5 coverage. 


\section{DNA Hybridization to NR@PDA@ssDNA}

A final set of measurements were then performed to confirm the bioactivity of the NR-ssDNA conjugates. Fig. 7(a) shows a series of bulk fluorescence measurements involving conjugates prepared with $\mathrm{P}_{\mathrm{NH} 2, \mathrm{Cy} 5}$ as well as $\mathrm{P}_{\mathrm{NH} 2}$ (i.e. no Cy5) and $\mathrm{T}_{30, \mathrm{NH} 2}$ control sequences for comparison. When various concentrations of $\mathrm{C}_{\mathrm{CY}_{3}} \mathrm{DNA}$ were added to each sample for a minimum of 3 hours before irradiating at $480 \mathrm{~nm}$, a smaller increase in Cy3 fluorescence emission associated with coupling between $\mathrm{Cy} 3$ and $\mathrm{Cy} 5$ was only observed for the NR-P $\mathrm{P}_{\mathrm{NH} 2, \mathrm{Cy} 5}$ conjugates. Furthermore, a net larger change in signal was observed for the conjugates prepared in the presence of $\mathrm{NaCl}$, highlighting the higher $\mathrm{P}_{\mathrm{NH} 2, \mathrm{Cy} 5}$ surface density. The control sequences were chosen to provide a clear demonstration that a Cy3-Cy5 interaction was occurring due to the formation of dsDNA rather than non-specific interactions or potential quenching of the Cy3 by PDA was responsible for the differences observed. For these bulk measurements, the overlap of the Cy5 emission with the LSPR of the NR's prevented this wavelength range from being accurately monitored. Consequently, we also performed additional single nanoparticle imaging measurements (see Fig. 7b) utilizing different combinations of $\mathrm{Cy} 3$ and $\mathrm{Cy} 5$ excitation and emission filters on the same sample and compared along-side a dark-field image for particle identification. This showed that Cy5 emission could be detected upon Cy3 excitation while the Cy3 emission was barely detectable under the same acquisition conditions, indicative of $\mathrm{Cy} 3-\mathrm{Cy} 5$ coupling and that a significant fraction of the surface immobilized ssDNA strands are available for hybridization.

The DNA conjugation and hybridization measurements together provide evidence of packed ssDNA monolayers forming NR@PSS@PDA substrates with good bioavailability. This was also supported with zeta-potential measurements, which typically demonstrated a change from c.a. -30 $\mathrm{mV}$ to $-15 \mathrm{mV}$ following DNA hybridization. In this initial study, we have chosen conditions aimed at maximizing the ssDNA surface loading density and allowing sufficient time during hybridization to ensure steady-state coverage. Future work exploring additional parameters such as ssDNA surface density, dye spacing from the PDA layer and monitoring in-situ surface hybridization kinetics will promote the utilization of this PDA surface chemistry for a wide range of nanoparticlebased sensing applications. 
(a)

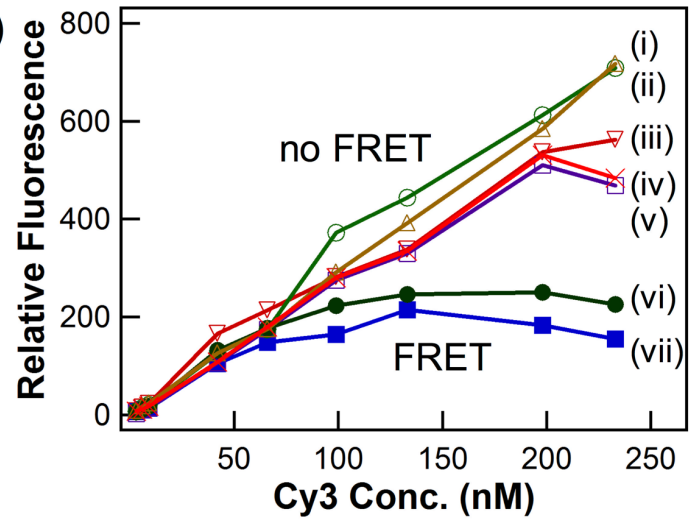

(b)
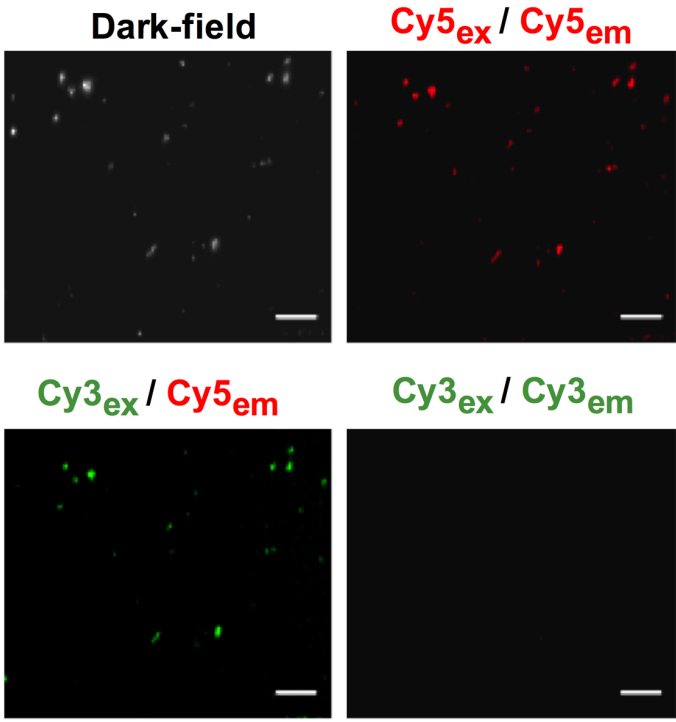

Fig. 7 Monitoring DNA ( $\left.C_{C Y 3}\right)$ hybridization on to NR-ssDNA conjugates. (a) Changes in Cy3 bulk solution fluorescence at added $C_{C Y 3}$ concentrations ranging from 3 to $233 \mathrm{nM}$ to: (i) $N R-T_{30, N H 2}$, (0.15 mM NaCl), (ii) NR-T30,NH2 only, (iii) $C_{C Y 3}$ only, (iv) NR- $P_{N H 2}$, (v) NR-P $P_{N H 2}(0.15 \mathrm{mM} \mathrm{NaCl}$ ), (vi) NR-P $P_{N H 2, C y 5}$ only, and (vii) NR- $P_{N H 2, C y 5},(0.15 \mathrm{mM} \mathrm{NaCl})$. Each data point corresponds to a minimum incubation time of at least 3 hours prior to bulk fluorescence measurement. (b) Correlated dark-field and fluorescence images of NR-P $P_{N H 2, C y 5}$ conjugates with different combinations of Cy3 and Cy5 excitation and emission filters. The NR conjugates were immobilized on a glass coverslip, which was subsequently incubated with 50 nM $C_{C y 3}$ in PBS buffer and then rinsed and dried prior to imaging. Scale bars $=10 \mu \mathrm{m}$.

\section{Conclusion}

In this article, we compared multiple routes for the controlled formation of polydopamine layers on gold nanorods with the subsequent aim of establishing a robust route for the fabrication of NR-ssDNA conjugates. While PDA chemistry has been frequently described as a universal coating and has been successfully demonstrated on a wide range of substrates, there have been no studies systematically looking at the role of surface functional groups and charge on the kinetics and thickness of the films deposited on nanoparticle or planar surfaces. The sensitivity of the nanorod LSPR peak combined with the ability to easily modify the NR surface via polyelectrolyte wrapping enabled us to reveal that the PDA layer formation is actually very sensitive to the properties of the underlying surface. A 
high loading density of ssDNA is critical for achieving the cooperative binding properties of DNAnanoparticle conjugates. While this has been well-studied on spherical nanoparticles, ${ }^{53}$ we hope that this work will promote the integration of DNA-anisotropic nanoparticle conjugates into more complex bionano systems. Furthermore, since antibodies and many other species can be readily conjugated to PDA, we expect the methodologies described here will further expand the utility of nanorods across multiple research areas.

\section{Acknowledgement}

This work has been supported by funding from mohe-casm, Ministry of Higher Education and Scientific Research, Egypt. AF is supported by the EPSRC and MRC Centre for Doctoral Training in Optical Medical Imaging (Ref: EP/ L016559/1). We also thank Dr K.A.H. Lau (University of Strathclyde) for valuable discussions. Research data associated with this paper will become available through the following link: http://doi.org/XXXXX.

\section{References}

1. X. Huang, S. Neretina and M. A. El-Sayed, Adv. Mater., 2009, 21, 4880-4910.

2. $\quad$ S. E. Lohse and C. J. Murphy, Chem. Mater., 2013, 25, 1250-1261.

3. C. Kinnear, H. Dietsch, M. J. D. Clift, C. Endes, B. Rothen-Rutishauser and A. Petri-Fink, Angew. Chem. Int. Ed., 2013, 52, 1934-1938.

4. A. McLintock, N. Hunt and A. W. Wark, Chem. Commun., 2011, 47, 3757-3759.

5. N. A. Merrill, M. Sethi and M. R. Knecht, ACS Appl. Mater. Interfaces, 2013, 5, 7906-7914.

6. N. D. Burrows, W. Lin, J. G. Hinman, J. M. Dennison, A. M. Vartanian, N. S. Abadeer, E. M. Grzincic, L. M. Jacob, J. Li and C. J. Murphy, Langmuir, 2016, 32, 9905-9921.

7. E. Locatelli, I. Monaco and M. Comes Franchini, RSC Advances, 2015, 5, 21681-21699.

8. C.-C. Chen, Y.-P. Lin, C.-W. Wang, H.-C. Tzeng, C.-H. Wu, Y.-C. Chen, C.-P. Chen, L.-C. Chen and Y.-C. Wu, J. Am. Chem. Soc., 2006, 128, 3709-3715.

9. J. H. Joo and J.-S. Lee, Anal. Chem., 2013, 85, 6580-6586.

10. A. Wijaya, S. B. Schaffer, I. G. Pallares and K. Hamad-Schifferli, ACS Nano, 2009, 3, 8086.

11. D. Shi, C. Song, Q. Jiang, Z.-G. Wang and B. Ding, Chem. Commun., 2013, 49, 2533-2535.

12. J. Li, B. Zhu, Z. Zhu, Y. Zhang, X. Yao, S. Tu, R. Liu, S. Jia and C. J. Yang, Langmuir, 2015, 31, 7869-7876.

13. Z. Li, Z. Zhu, W. Liu, Y. Zhou, B. Han, Y. Gao and Z. Tang, J. Am. Chem. Soc., 2012, 134, 3322-3325.

14. W. Ma, H. Kuang, L. Xu, L. Ding, C. Xu, L. Wang and N. A. Kotov, Nature Commun., 2013, 4, 2689.

15. S. H. Baek, A. W. Wark and H. J. Lee, Anal. Chem., 2014, 86, 9824.

16. A. Wijaya and K. Hamad-Schifferli, Langmuir, 2008, 24, 9966-9969.

17. V. Baumann, P. J. Friedrich Rottgermann, F. Haase, K. Szendrei, P. Dey, K. Lyons, R. Wyrwich, Gra, J. Stehr, L. Ullerich, F. Bursgens and J. Rodriguez-Fernandez, RSC Advances, 2016, 6, 103724-103739.

18. I. E. Sendroiu, M. E. Warner and R. M. Corn, Langmuir, 2009, 25, 11282-11284.

19. D. Pissuwan and T. Niidome, Nanoscale, 2015, 7, 59-65.

20. L. G. Xu, Y. Liu, Z. Y. Chen, W. Li, L. M. Wang, X. C. Wu, Y. L. Ji, Y. L. Zhao, L. Y. Ma, Y. M. Shao and C. Y. Chen, Nano Lett., 2012, 12, 2003-2012. 
21. H. Lee, S. M. Dellatore, W. M. Miller and P. B. Messersmith, Science, 2007, 318, 426-430.

22. M. Liu, G. Zeng, K. Wang, Q. Wan, L. Tao, X. Zhang and Y. Wei, Nanoscale, 2016, 8, 16819-16840.

23. Y. H. Ding, M. Floren and W. Tan, Biosurface and Biotribology, 2016, 2, 121-136.

24. H. O. Ham, Z. Liu, K. H. A. Lau, H. Lee and P. B. Messersmith, Angew. Chem. Int. Ed., 2011, 50, 732-736.

25. J. B. Wood, M. W. Szyndler, A. R. Halpern, K. Cho and R. M. Corn, Langmuir, 2013, 29, 10868-10873.

26. C. Wang, J. Zhou, P. Wang, W. He and H. Duan, Bioconjugate Chem., 2016, 27, 815-823.

27. C. K. K. Choi, J. Li, K. Wei, Y. J. Xu, L. W. C. Ho, M. Zhu, K. K. W. To, C. H. J. Choi and L. Bian, J. Am. Chem. Soc., 2015, 137, 7337-7346.

28. N. Orishchin, C. C. Crane, M. Brownell, T. Wang, S. Jenkins, M. Zou, A. Nair and J. Chen, Langmuir, 2017, 33, 6046-6053.

29. J. Zhou, B. Duan, Z. Fang, J. Song, C. Wang, P. B. Messersmith and H. Duan, Adv. Mater., 2014, 26, 701-705.

30. J. Zhou, Q. Xiong, J. Ma, J. Ren, P. B. Messersmith, P. Chen and H. Duan, ACS Nano, 2016, DOI: 10.1021/acsnano.6b05951.

31. W. Zhang, F. Wang, Y. Wang, J. Wang, Y. Yu, S. Guo, R. Chen and D. Zhou, J. Controlled Release, 2016, 232, 9-19.

32. K. C. L. Black, J. Yi, J. G. Rivera, D. C. Zelasko-Leon and P. B. Messersmith, Nanomedicine, 2013, 8, 17-28.

33. S. Wang, X. Zhao, S. Wang, J. Qian and S. He, ACS Appl. Mater. Interfaces, 2016, 8, 24368-24384.

34. L. Zhang, H. Su, J. Cai, D. Cheng, Y. Ma, J. Zhang, C. Zhou, S. Liu, H. Shi, Y. Zhang and C. Zhang, ACS Nano, 2016, 10, 10404-10417.

35. K. C. L. Black, T. S. Sileika, J. Yi, R. Zhang, J. G. Rivera and P. B. Messersmith, Small, 2014, 10, 169-178.

36. K. Kang, H. Jang and Y.-K. Kim, Analyst, 2017, 142, 2372-2377.

37. C. A. Cunha-Matos, O. R. Millington, A. W. Wark and M. Zagnoni, Lab on a Chip, 2016, 16, 3374-3381.

38. B. Nikoobakht and M. A. El-Sayed, Chem. Mater., 2003, 15, 1957-1962.

39. C. J. Orendorff and C. J. Murphy, J. Phys. Chem. B, 2006, 110, 3990-3994.

40. A. McLintock, C. A. Cunha-Matos, M. Zagnoni, O. R. Millington and A. W. Wark, ACS Nano, 2014, 8, 8600-8609.

41. A. Gole and C. J. Murphy, Chem. Mater., 2005, 17, 1325-1330.

42. P. K. Jain, S. Eustis and M. A. El-Sayed, J. Phys. Chem. B, 2006, 110, 18243-18253.

43. L. Wang, Y. Zhu, L. Xu, W. Chen, H. Kuang, L. Liu, A. Agarwal, C. Xu and N. A. Kotov, Angew. Chem. Int. Ed., 2010, 49, 5472-5475.

44. Y. Liu, K. Ai and L. Lu, Chem. Rev., 2014, 114, 5057-5115.

45. V. Ball, D. D. Frari, V. Toniazzo and D. Ruch, J. Colloid Interface Sci., 2012, 386, 366-372.

46. G. J. Nusz, A. C. Curry, S. M. Marinakos, A. Wax and A. Chilkoti, ACS Nano, 2009, 3, 795-806.

47. A. McLintock, H. J. Lee and A. W. Wark, Phys. Chem. Chem. Phys., 2013, 15, 1883518843.

48. W. H. Ni, Z. Yang, H. J. Chen, L. Li and J. F. Wang, J. Am. Chem. Soc., 2008, 130, 66926693.

49. H. Lee, J. Rho and P. B. Messersmith, Adv. Mater., 2009, 21, 431-434.

50. J. Yang, M. A. Cohen Stuart and M. Kamperman, Chem. Soc. Rev., 2014, 43, 8271-8298.

51. S. J. Hurst, A. K. R. Lytton-Jean and C. A. Mirkin, Anal. Chem., 2006, 78, 8313-8318.

52. H. D. Hill, J. E. Millstone, M. J. Banholzer and C. A. Mirkin, ACS Nano, 2009, 3, 418-424.

53. J. I. Cutler, E. Auyeung and C. A. Mirkin, J. Am. Chem. Soc., 2012, 134, 1376-1391. 


\section{TOC Image}

The formation of a stable polydopamine layer on a nanorod surface depends on the underlying chemistry and optimization enables the formation of packed ssDNA monolayers for bioaffinity applications.

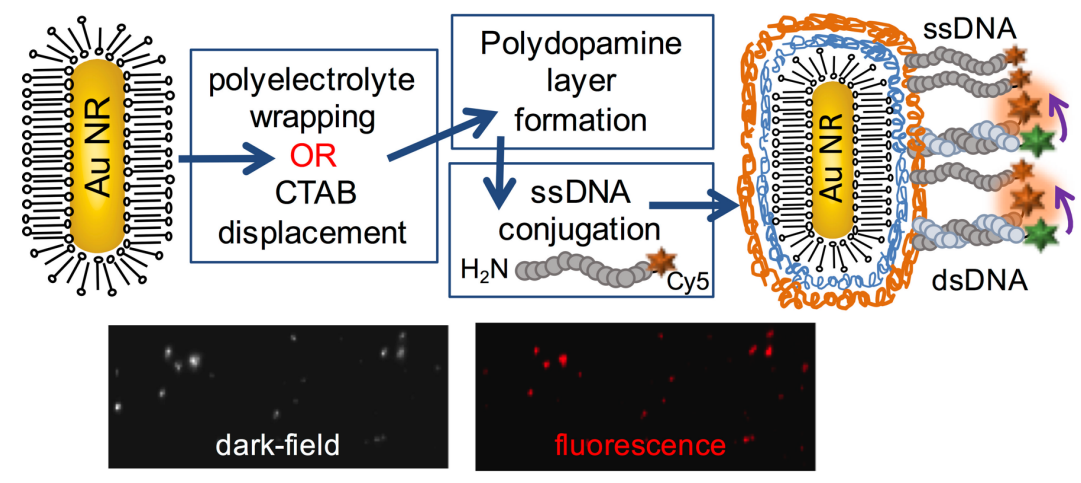

